\title{
Asynchronous Hybrid Deep Learning (AHDL): A Deep Learning Based Resource Mapping in DVFS Enabled Mobile MPSoCs
}

\author{
Somdip Dey \\ Engineering \& Research \\ Nosh Technologies \\ U.K. \\ dey@nosh.tech; somdip.dey@essex.ac.uk
}

\author{
Suman Saha \\ Engineering \& Research \\ Nosh Technologies \\ U.K. \\ suman.saha@nosh.tech
}

\author{
Amit Singh \\ CSEE \\ University of Essex \\ U.K. \\ a.k.singh@essex.ac.uk
}

\author{
Klaus McDonald-Maier \\ CSEE \\ University of Essex \\ U.K.
}

kdm@essex.ac.uk

\begin{abstract}
Mapping resources to tasks accurately in order to gain performance, energy efficiency, reduction in peak temperature, etc. on an embedded/Edge device is a big challenge. Machine learning has proven to be effective in learning heuristics based resource mapping approaches, but its success is bound by the quality of feature extraction. Additionally, feature extraction in such approaches not just requires expert domain knowledge and human effort, but at the same time requires the application (tasks) to be profiled for such processes. Therefore, the efficacy of such resource mapping methodologies depends on expertise, skills, profiling time and architecture of the system. In this paper, we propose a novel methodology, Asynchronous Hybrid Deep Learning (AHDL), which sets a new paradigm of using Deep Learning approaches to map resources to application (tasks). In our approach, we leverage task profiling methodologies to achieve accurate mapping in order to achieve greater reward from the system, but at the same time is able to allocate resources to unprofiled application (tasks) at the same time without the need of manual feature extraction by domain experts. Our proposed methodology is able to achieve competitive results in comparison with the state-of- the-art without the usual associated challenges such as manual feature extraction.
\end{abstract}

Index Terms-DVFS, MPSoC, asynchronous hybrid deep learning, resource mapping

\section{IntRoduction AND Motivation}

Recently we could see an increase in embedded/Edge computing systems [1], which employs heterogeneous multiprocessor systems-on-chips (MPSoCs) consisting of different types of processing cores such as CPU and GPU. These architectures provide opportunities to exploit distinct features of CPU and GPU cores to meet performance, power consumption, thermal behaviour and security requirements. Furthermore, the cores in these MPSoCs support dynamic voltage and frequency scaling (DVFS) [2]-[5], which can be used to reduce dynamic power consumption $\left(P \propto V^{2} f\right)$ [6], [7]. This helps to reduce the power consumption by executing the workload over extra time at a lower voltage and frequency, which could be accounted for reduced power consumption.

On the other hand, several studies, which are focused on extracting features from the source code of an application and

This work was supported by Nosh Technologies under Grant nosh/agritech-000001 and by the U.K. Engineering and Physical Sciences Research Council (EPSRC) under Grant EP/P017487/1, Grant EP/R02572X/1, Grant EP/P016006/1 and Grant EP/V000462/1 then utilizing several machine learning models [8]-[13] such as Support Vector Machines (SVMs), Nearest Neighbor, etc. to classify different set of applications and then deciding the resources that need to be allocated to such applications. Using such methodologies also have their own disadvantages. Depending on feature extraction such as number of code blocks, branches, divergent instructions, and then utilizing machine learning on them usually requires accurate identification of features from the training data and then feeding them to the model. Extracting features from a source code of a program and then feeding to the machine learning model so that further inference could be made is difficult in many ways and often requires human intervention (domain experts). One of the key challenges in this area is to automate such feature extraction process from the source code such that appropriate computing resources could be allocated to the associated application to reduce power consumption and peak temperature of the device.

In this paper, we propose a novel method to allocate resources to application (tasks) without the need of manual feature extraction by domain experts. We leverage Deep Learning [14] approach to classify different types of applications and then allocate computing resources for the particular application to improve performance, power consumption, thermal behaviour and security of the device, as required. To this extent, the concrete contribution of this paper are as follows.

1) Propose a method for resource allocation to application (tasks) without the need of manual feature extraction by domain experts.

2) Experimental results on real hardware device (Odroid XU4 [15]).

3) Comparative study of the proposed approach with the state-of-the-art methods.

\section{RELATED WORK}

In several earlier studies [16]-[20], many researchers have focused on designing methodologies and frameworks to optimize power and operating temperature of MPSoCs based on different approaches to computing resource mapping. One such noteworthy study is performed by Ghasemazar et al. [16] where the researchers proposed a hierarchical framework leveraging DVFS capabilities of the processing cores to find 
the optimal voltage-frequency to cater for power consumption and temperature. This methodology was successful in achieving $20 \%$ performance boost without impacting the overall operating temperature but their experiments focused mainly on CISC architectures and all results were based on a MATLABbased Chip Multiprocessor simulator. In another paper [17], Kamal et al. proposed a heuristic based thermal stress-aware mechanism for management of power and temperature in MPSoCs formulated in a convex optimization problem. This approach was implemented on Sniper multicore simulator [18] and was able to reduce spatial and temporal thermal gradients by $7 \%$ and $18 \%$ respectively when compared to the work in [16].

In [19] Sigla et al. present a predictor using power sensors to predict the next power consumption based on the following frequency setting is developed. Their technique uses a leakage power model of the ARM's big.LITTLE architecture on the Odroid-XU3 to validate its predictor and Dynamic Power and Frequency Management technique. An Extension of this work has also been published in [20]. Both [19], [20] methods involve predicting the future core temperatures to adjust the workloads or frequencies before exceeding a set threshold. In [21], Reddy et al. proposed a Memory Reads Per Instruction (MRPI) metric based computing resource mapping, whereas, in [2], Dey et al. proposed a methodology to optimize temperature by performing DVFS on CPUs based on the relationship between operating frequency of the processing cores and temperature of the MPSoC.

Moreover, none of the aforementioned approaches [16]-[20] optimize the power and thermal behaviour of the MPSoC by optimizing the computing resource as required by the type of executing applications. Executing applications could potentially fall into any of the following three categories [22]: Compute intensive, Memory intensive \& Mixed workload, where the application is both Compute and Memory intensive. Additionally, executing application could also require different processing cores such as CPU and GPU at different times of the execution. The aforementioned approaches does not account for such changing demand in processing elements based on the type of the executing application.

The primary focus of this work is to design an approach to allocate computing resources to application/tasks by extracting features of executing applications automatically such that performance, power and temperature of the MPSoC could be optimized.

\section{Proposed Methodology: Asynchronous HYBRID DEEP LEARNING (AHDL)}

To overcome the challenge of mapping computing resources $(M)$ to new tasks or applications, which might or might not have been profiled or executed before, we propose an Asynchronous Hybrid Deep Learning (AHDL) resource mapping approach, which determines (classify) the type of executing application (tasks) without requiring manual feature extraction and then allocates the appropriate computing resources based on that. AHDL has two phases: Learning Phase and Decision Phase. To get more accurate resource mapping in order to achieve the most from the system we use offline profiling of the application instance $\left(A p p_{i}\right)$. We refer our desired output requirement of the $A p p_{i}$ on the system as Reward $(R)$. Our goal is to maximize the Reward of $A p p_{i}$ while minimizing the resources being allocated to $A p p_{i}$. Reward will vary depending on the application and the user's need. For example, if we are trying to map resources for a face detection application on the MPSoC where performance of the application is crucial in terms of frames per second ( $f p s$ ) for the recorded video then $f$ ps becomes the Reward in this case. Whereas in another example where we are trying to map resources to an instance of audio decoding application then execution time $E T_{A p p_{i}}$ of the application becomes the Reward, which we need to prioritize to maximize. Therefore, if $M$ is the resources that need to be allocated to the application $A p p_{i}, P E s$ is the set of all available processing elements in the system, $F_{P E s}$ is the set of all associated frequency scaling levels leveraging DVFS capabilities for all processing elements in $P E s$, the main objective of the AHDL resource mapping approach is as follows:

$$
\forall M \in\left\{P E s, F_{P E s}\right\}: \operatorname{maximize}(R) \propto \operatorname{minimize}(M)
$$

Note: For some cases, the main objective of AHDL resource mapping could be to minimize the value of reward $(R)$ instead while minimizing the resources to be allocated. For example, if an application is utilizing data encryption algorithm then instead of focusing on performance the main objective should be to reduce the operating temperature of the processing elements in order to protect against temperature side-channel attacks. Hence, in this case the Reward will be temperature and the goal is to minimize $R$ while minimizing the resources being allocated to the application $A p p_{i}$. Therefore, the main objective of the AHDL could be modified to represent as follows:

$$
\forall M \in\left\{P E s, F_{P E s}\right\}: \operatorname{minimize}(R) \propto \operatorname{minimize}(M)
$$

\section{A. Overview of $A H D L$}

For every instance of an application $\left(A p p_{i}\right)$ from a set of applications (Apps), App $i$ is profiled in the Regression Module in Training Phase, where frequency of the processing elements $(P E s)$ are modified along with the number of processing elements $(P E)$ mapped to the task(s) to generate a set of Rewards $(S(R))$. From this set of Rewards $(S(R))$, the maximum of the set is chosen to reflect the maximized Reward and the associated mapped process elements, associated frequencies and other relationship variables are saved on the memory. These are the Reward Profiled Data, which will be used during the Decision Phase to map proper resources along with associated frequencies of the processing elements.

Back in the Training Phase, the source code of $A p p_{i}$ is fed to Imager Module, which is a software agent that converts the source code into visual images such that the image $\left(I_{A p p_{i}}\right)$ representing the source code of the application $A p p_{i}$ could be used to train a pre-Trained convolutional neural network (CNN) to understand the difference between different set of applications. After the training of the $\mathrm{CNN}$ is complete, the 
trained CNN (Coder CNN) could be used to classify an application if that application is not profiled before. Using this approach we could classify new applications during runtime and map appropriate resources to this application based on our heuristics in the Decision Phase. More details on each phases (Training \& Decision) and modules (Regression \& Visual Convolutional Neural Network) are provided in the following subsections.

\section{B. Training Phase}

The Training Phase is performed completely offline due to the time required for profiling the applications (Apps). In this Phase we take an application instance $A p p_{i}$ from a set of applications Apps and pass it through a Regression Module. We call this as Regression Module because we use simple linear regression to evaluate and set the frequency levels of the processing elements in order to achieve the maximum Reward. But before utilizing this approach, the type of Reward is required to be finalized by the user based on the application. For example, if the application is a computer vision one then frames per second is chosen as the Reward, whereas for execution critical application the execution time is chosen as a Reward. Once the Reward is finalized, we profile $A p p_{i}$ in the Regression Module.

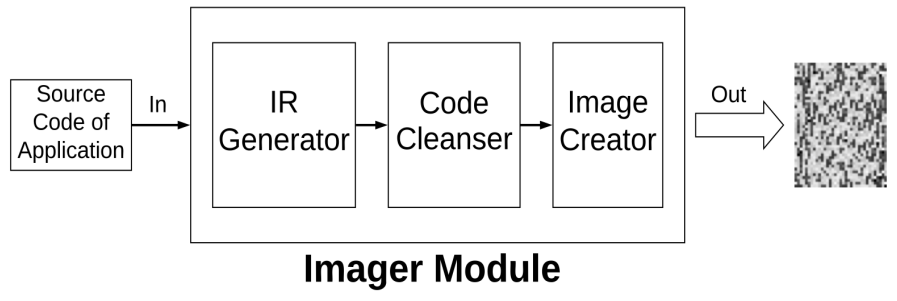

Fig. 1. Block digram of Imager Module

1) Regression Module: If we assume that a processing element $P E_{i}$ has an operating frequency of $f_{P E_{i}}$ and utilizing the concept of DVFS we could derive the governing equation (see Eq. 3) for our regression model as follows:

$$
R=\alpha f_{P E_{i}}+\beta
$$

In Eq. $3 \alpha$ is the relationship variable whereas $\beta$ is the intercept. Now, for MPSoCs which allow cluster wide DVFS capabilities with a cluster of processing elements having the same operating frequency $\left(f_{P E_{\text {cluster }}}\right)$ instead of having individual operating frequencies $\left(f_{P E_{i}}\right)$ for each $\mathrm{PE}$, the equation could be written as follows.

$$
R=\alpha f_{P E_{\text {cluster }}}+\beta
$$

If we consider that $F_{P E s}$ is the set of all the possible frequencies at which the processing elements could operate and $S(R)$ be the set of all associated Rewards for each operating frequency in $F_{P E s}$ and $R_{\text {desired }}$ be the desired maximum Reward we want to achieve then we get the governing equation as follows:

$$
\forall f_{P E_{i}}, f_{P E_{\text {cluster }}} \in F_{P E s}: R_{\text {desired }}=\max S(R)
$$

Therefore, using the Eq. 3, 4 and 5 we profile $A p p_{i}$ and save the number of PEs, associated operating frequencies, $\alpha$ and $\beta$ on the memory. We then repeat this approach to profile the same $A p p_{i}$ for $M$ number of times to achieve a Reward $\left(\right.$ Reward $\left._{\text {desired }_{i}}\right)$ for each instance and use ensemble average approach [23] to get the desired Reward $\left(R_{\text {desired }}^{\text {App }}\right.$ ) for the application. The mathematical representation of this could be fulfilled by Eq. 6 .

$$
R_{\text {desired }^{\text {App }}}=\frac{1}{M} \times \sum_{i=1}^{M} R_{\text {desired }_{i}}
$$

The output of Eq. 6 (see Fig. 2) are saved as the Reward Profiled Data, which would be later used in the Decision Phase. Now, the program source code of $A p p_{i}$ is fed to our Imager Module of Visual Convolutional Neural Network Module, which is the main software agent aiding in representing the program source code into an image so that it could be used by a visual CNN for automatic feature extraction method without requiring skilled manual feature extraction.

2) Visual Convolutional Neural Network Module: This module is responsible for converting program source code into images and train a chosen pre-Trained CNN using Transfer Learning such that the $\mathrm{CNN}$ is capable of extracting features automatically from images. This approach was first proposed in [22] by Dey et al.

Imager Module: Fig. 1 shows the inner working of the Imager Module and the algorithm is provided in Algo. 1. The Imager Module is a software agent where the program source code is fed and the agent first converts the source code to an optimized LLVM intermediate representation (IR) [24]. In the Imager Module, a sub module named Code Cleanser then acts on the LLVM IR to strip off all the unwanted codes such as metadata and comments and later processed by another sub module named Image Creator to be represented as an image $\left(I_{A p p_{i}}\right)$. In the Image Creator sub module, each byte of cleansed LLVM IR is read as an integer value, which ranges from 0 to 255 (ASCII value). Each of these bytes are then represented as a pixel value in a $w \times h$ image where $w$ is the width and $h$ is the height of the image. If there are $n$ number of bytes of cleansed LLVM IR code then $n, w \& h$ form the relationship of $n=w \times h$.

After the creation of image representation by Imager Module, $I_{A p p_{i}}$ of several different applications (Apps) is then categorized into different classes so that the visual CNN could be trained for future classification and further decision making based on our heuristics. If we consider $I_{A p p s}$ as the dataset of images representing all the source codes of Apps then we run the dataset $I_{A p p s}$ through the pre-Trained $\mathrm{CNN}$ and training a new, randomly initialized classifier on top of the semantic image output vector using the concept of Transfer Learning in CNN [25], [26]. After training the CNN with our own custom classifier we call the CNN as Coder $C N N$. Note: Categorizing each $I_{A p p_{i}}$ would depend on the choice of the user and the object of maximizing the Reward. For example, based on the types of applications being profiled we segregated the image representation into 3 categories: compute intensive, memory intensive and mixed load (both compute 


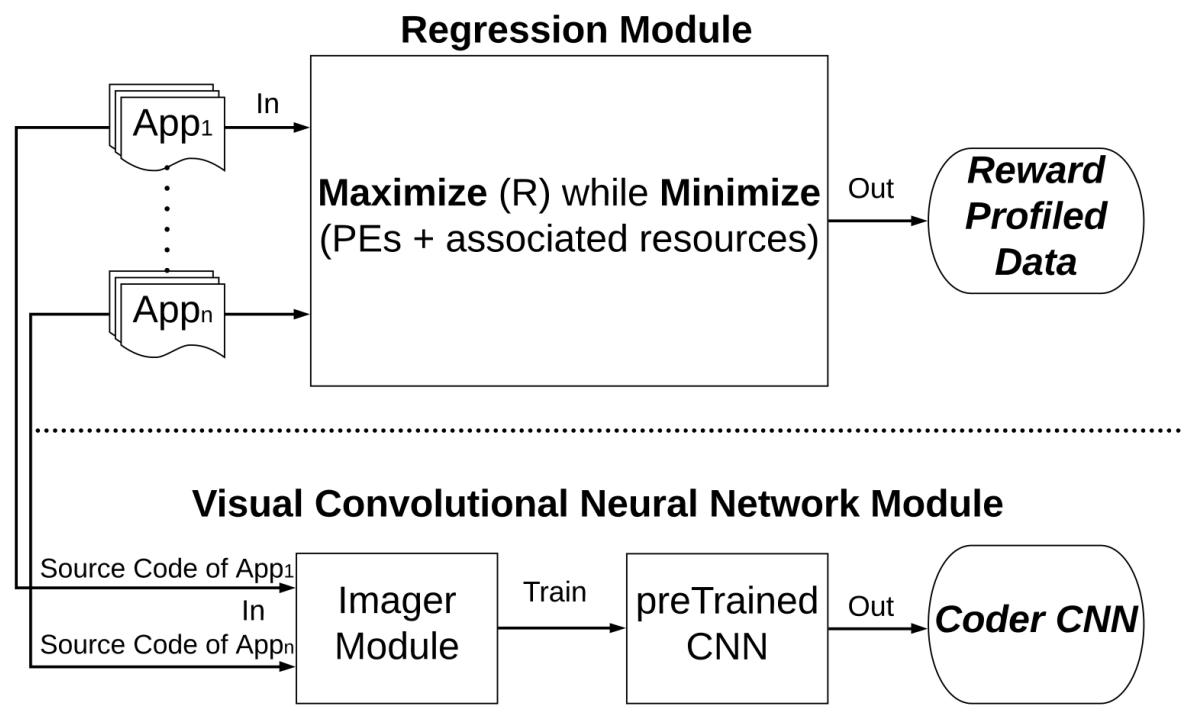

Fig. 2. Block digram of Training Phase of Asynchronous Hybrid Deep Learning Resource Mapping

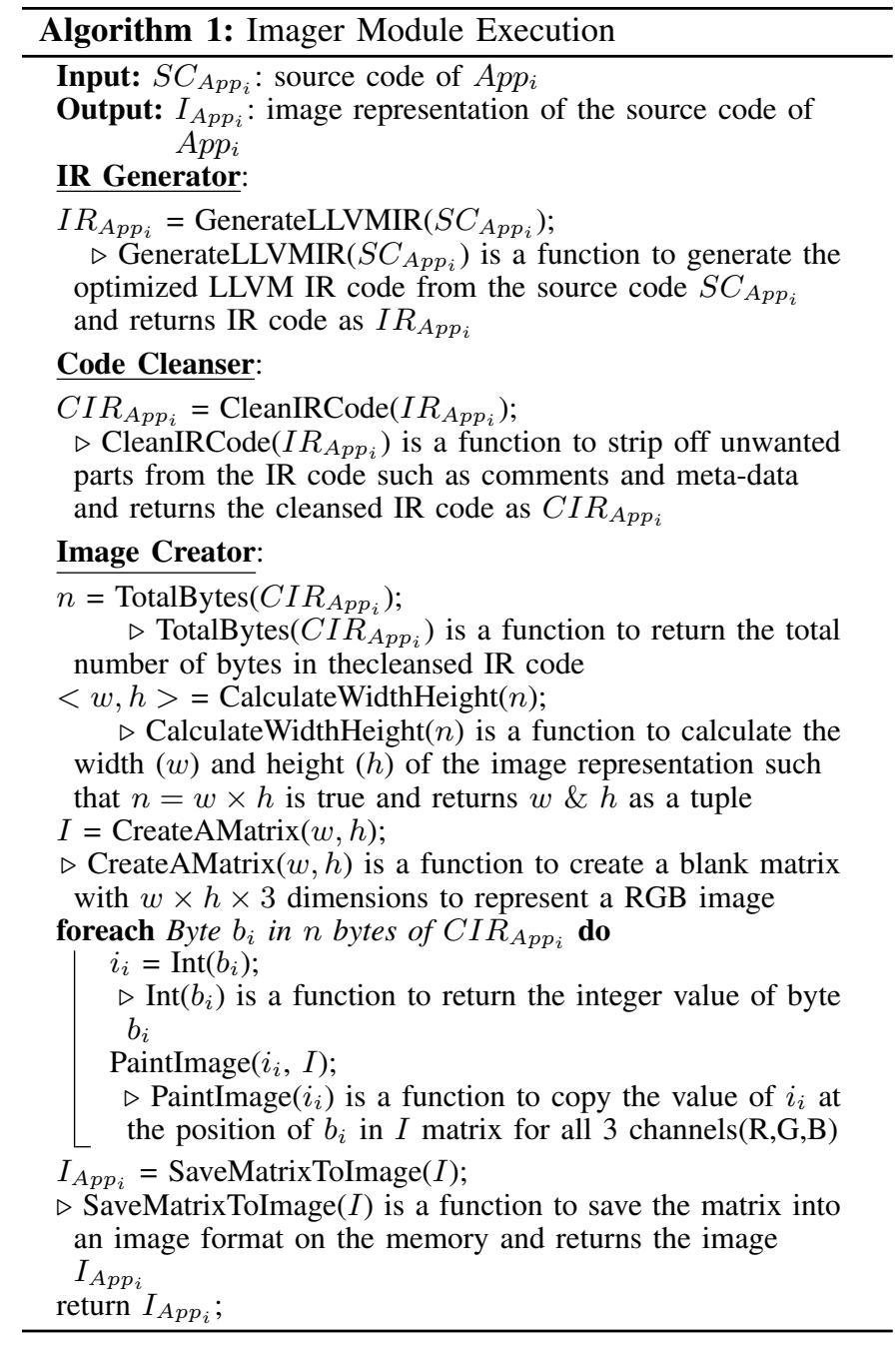

and memory intensive) [22]. We chose these 3 categories because we allocate resources to new applications based on their computational capacities in the Decision Phase.

\section{Decision Phase}

The block diagram of Decision Phase is provided in Fig. 3. The knowledge gathered through profiling (Reward Profiled Data \& Coder CNN) from the Training Phase is transfered on the MPSoC. Decision Phase is implemented as a software agent. When an application instance $A p p_{i}$ is executed on the MPSoC, the agent first checks whether $A p p_{i}$ is profiled in advance in the Training Phase. If no associated Reward Profiled Data on $A p p_{i}$ is found then the source code of the application $A p p_{i}$ is fed to the Imager Module to create the image representation $I_{A p p_{i}}$ so that it could be classified by the Coder $C N N$ and then allocation of resources take place based on the classification. But if the associated Reward Profiled Data on $A p p_{i}$ is found then resources are allocated based on the Reward Profiled Data. We have to keep in mind that resource allocation through Regression Module of Decision Phase is more accurate because the application has been profiled in advance but the resource allocation achieved from the Visual Convolutional Neural Network Module in the Decision Phase is less accurate but has been able to produce competitive results to achieve maximum Reward when compared to the state-of-the-art (see Sec. IV for comparison) without requiring any manual feature selection of the application. Note: For Visual Convolutional Neural Network Module we are setting different resources (operating frequency and number of processing elements such as big and LITTLE CPUs) for different types of applications. The following resources are chosen for different types of applications for our experimental platform (Odroid XU4 [15]): compute intensive (all the big CPUs, all the LITTLE CPUs, maximum operating frequency of the big CPUs + LITTLE CPUs), memory intensive (half of the total number of LITTLE CPUs, maximum operating frequency of the LITTLE CPUs) and mixed load (half of the total number 


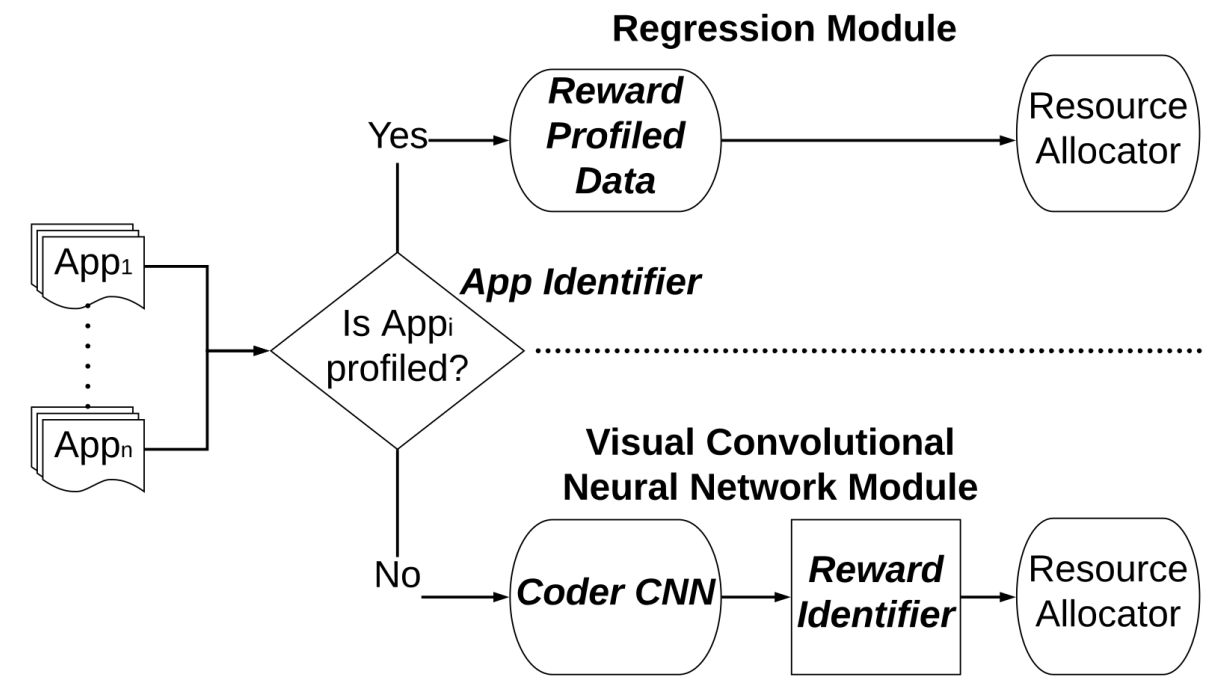

Fig. 3. Block digram of Decision Phase of Asynchronous Hybrid Deep Learning Resource Mapping

of the big CPUs, all the LITTLE CPUs, middle operating frequency of the big CPUs + LITTLE CPUs).

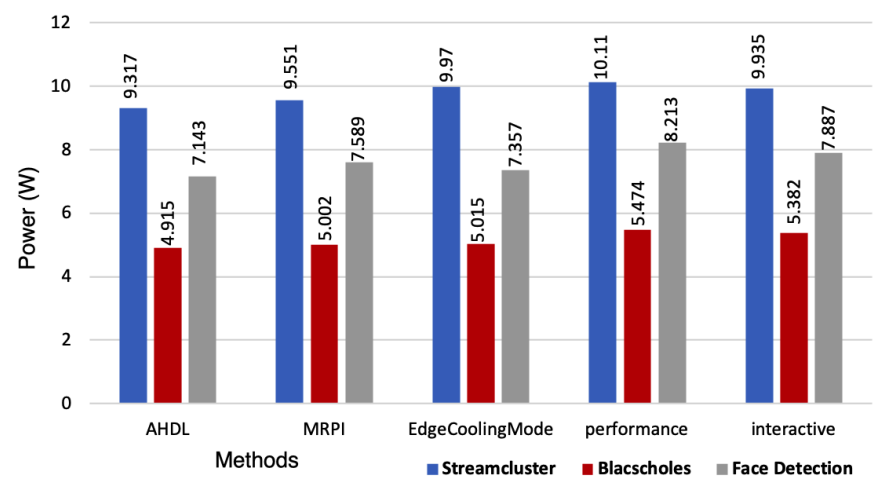

(a) Power consumption

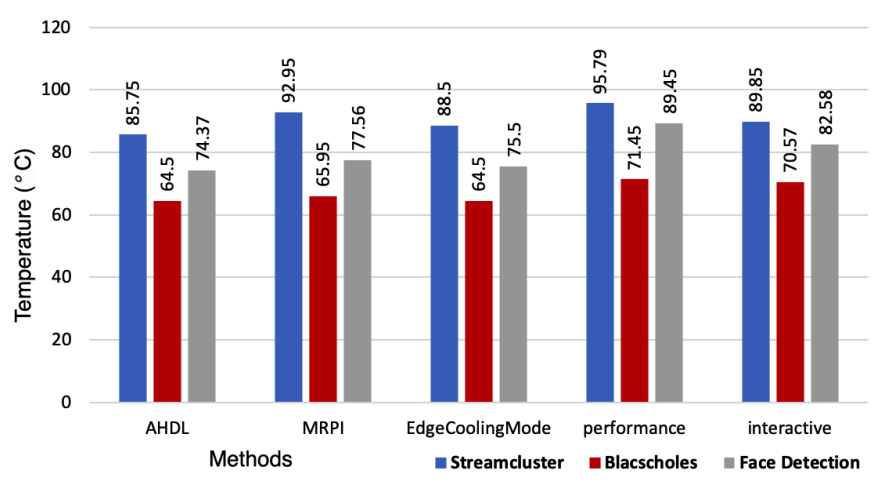

(b) Peak temperature

Fig. 4. Comparative study of power consumption and peak temperature for different methods on different benchmark applications

\section{EXPERIMENTAL RESULTS}

\section{A. Hardware \& Software Infrastructure}

1) Hardware Infrastructure: Nowadays heterogeneous MPSoCs consist of different types of cores, either having the same or different instruction set architecture (ISA). Moreover, the number of cores of each type of ISA can vary based on MPSoCs and are usually clustered if the types of cores are similar. For this research, we have chosen an Asymmetric Multicore Processors (AMPs) system-on-chip (AMPSoC), which is a special case of heterogeneous MPSoC and has clustered cores on the system. Our study was pursued on the Odroid XU4 board [15], which employs the Samsung Exynos 5422 [27] MPSoC. Exynos 5422 is based on ARM's big.LITTLE technology [28] and contains cluster of 4 ARM Cortex-A15 (big) CPU cores and another of 4 ARM Cortex-A7 (LITTLE) CPU cores, where each core implements the ARM v7A ISA. This MPSoC provides dynamic voltage frequency scaling feature per cluster, where the big core cluster has 19 frequency scaling levels, ranging from $200 \mathrm{MHz}$ to $2000 \mathrm{MHz}$ with each step of $100 \mathrm{MHz}$ and the LITTLE cluster has 13 frequency scaling levels, ranging from $200 \mathrm{MHz}$ to $1400 \mathrm{MHz}$, with each step of $100 \mathrm{MHz}$. Additionally, each core on the cluster has a private L1 instruction and data cache, and a L2 cache, which is shared across all the cores within a cluster.

Since Odroid XU4 board does not have an internal power sensor onboard, hence an external power monitor [29] with networking capabilities over WIFI is used to take power consumption readings. On the Odroid XU4 the temperature sensors are located on the big CPUs.

2) Software Infrastructure: For multi-core systems, multithreaded applications are heavily used in recent times to represent workloads as they could leverage concurrency and parallel processing. Examples of such applications are available in several benchmarks such as PARSEC [30]. For our experiments we chose Streamcluster with native option and Blackscholes with native option from the PARSEC benchmarks' suit because these applications closely represented realworld mixed load applications. We also validated our approach for face detection using Haar-cascade [31] application [32]. For our experiments each benchmark applications were executed five times and the average power consumption and peak 
temperature of the big CPUs are reported in the results. For Streamcluster and Blackscholes the Reward (desired output) was chosen to be the least execution time, whereas, for the face detection application 30 FPS was chosen to be the Reward (desired output) of AHDL method. We have run all our experiments on UbuntuMate version 14.04 (Linux Odroid Kernel: 3.10.105).

\section{B. Results and comparative study}

We evaluated our AHDL approach with state-of-the-art methods such as Memory Reads Per Instruction (MRPI) by Reddy et al. [21] and EdgeCoolingMode by Dey et al. [2] along with default Linux governors such as performance and interactive. Fig. IV.(a) shows the comparative study for the average power consumption between the different methods including AHDL for the benchmark applications, whereas, Fig. IV.(b) shows the comparative study for the average peak temperature of the big CPUs for the different methods. From Fig. IV we can notice that AHDL outperforms the state-ofthe-art methods and achieves reduced power consumption and peak temperature.

\section{CONCLUSION}

In this paper, we proposed Asynchronous Hybrid Deep Learning (AHDL) resource mapping approach, which classifies the type of executing application (tasks) without requiring manual feature extraction and then allocates the appropriate computing resources based on that to achieve reduced power consumption and peak temperature in embedded mobile devices. Experimental results on real hardware platform, Odroid $\mathrm{XU} 4$, proves the efficacy of the proposed approach while being competitively better than the state-of-the-art methods.

\section{REFERENCES}

[1] S. Dey et al., "Energy efficiency and reliability of computer vision applications on heterogeneous multi-processor systems-on-chips (mpsocs)."

[2] — "Edgecoolingmode: An agent based thermal management mechanism for dvfs enabled heterogeneous mpsocs," in VLSI Design and 18th International Conference on Embedded Systems, 2019. 32nd International Conference on. IEEE, 2019.

[3] S. Dey, A. K. Singh, and K. D. McDonald-Maier, "P-edgecoolingmode: an agent-based performance aware thermal management unit for dvfs enabled heterogeneous mpsocs," IET Computers \& Digital Techniques, vol. 13, no. 6, pp. 514-523, 2019.

[4] S. Dey, A. K. Singh, X. Wang, and K. D. McDonald-Maier, "Deadpool: Performance deadline based frequency pooling and thermal management agent in dvfs enabled mpsocs," in 2019 6th IEEE International Conference on Cyber Security and Cloud Computing (CSCloud)/2019 5th IEEE International Conference on Edge Computing and Scalable Cloud (EdgeCom). IEEE, 2019, pp. 190-195.

[5] A. K. Singh, S. Dey, K. R. Basireddy, K. McDonald-Maier, G. V. Merrett, and B. M. Al-Hashimi, "Dynamic energy and thermal management of multi-core mobile platforms: A survey," IEEE Design \& Test, 2020.

[6] S. Isuwa, S. Dey, A. K. Singh, and K. McDonald-Maier, "Teem: Online thermal-and energy-efficiency management on cpu-gpu mpsocs," in 2019 Design, Automation \& Test in Europe Conference \& Exhibition (DATE). IEEE, 2019, pp. 438-443.

[7] S. Dey, A. K. Singh, S. Saha, X. Wang, and K. D. McDonaldMaier, "Rewardprofiler: A reward based design space profiler on dvfs enabled mpsocs," in 2019 6th IEEE International Conference on Cyber Security and Cloud Computing (CSCloud)/2019 5th IEEE International Conference on Edge Computing and Scalable Cloud (EdgeCom). IEEE, 2019, pp. 210-220.

[8] B. Taylor, V. S. Marco, and Z. Wang, "Adaptive optimization for opencl programs on embedded heterogeneous systems," ACM SIGPLAN Notices, vol. 52, no. 5, pp. 11-20, 2017.
[9] C. Cummins, P. Petoumenos, Z. Wang, and H. Leather, "End-to-end deep learning of optimization heuristics," in 2017 26th International Conference on Parallel Architectures and Compilation Techniques (PACT). IEEE, 2017, pp. 219-232.

[10] S. Dey, S. Saha, A. Singh, and K. McDonald-Maier, "Fruitvegcnn: Power-and memory-efficient classification of fruits \& vegetables using cnn in mobile mpsoc," in 2020 IEEE 17th India Council International Conference (INDICON). IEEE, 2020, pp. 1-7.

[11] S. Dey, A. K. Singh, D. K. Prasad, and K. McDonald-Maier, "Temporal motionless analysis of video using cnn in mpsoc," in 2020 IEEE 31st International Conference on Application-specific Systems, Architectures and Processors (ASAP). IEEE, 2020, pp. 73-76.

[12] S. Dey, A. K. Singh, D. K. Prasad, and K. D. Mcdonald-Maier, "Ironman: An approach to perform temporal motionless analysis of video using cnn in mpsoc," IEEE Access, vol. 8, pp. 137 101-137 115, 2020.

[13] S. Dey et al., "User interaction aware reinforcement learning for power and thermal efficiency of cpu-gpu mobile mpsocs," in 2020 DATE. IEEE, 2020

[14] Y. LeCun, Y. Bengio, and G. Hinton, "Deep learning," nature, vol. 521, no. 7553 , p. 436,2015

[15] “Odroid-xu4," https://goo.gl/KmHZRG, accessed: 2018-07-23.

[16] M. Ghasemazar, H. Goudarzi, and M. Pedram, "Robust imization of a chip multiprocessor's performance under power and thermal constraints," in Computer Design (ICCD), 2012 IEEE 30th International Conference on. IEEE, 2012, pp. 108-114.

[17] M. Kamal, A. Iranfar, A. Afzali-Kusha, and M. Pedram, "A thermal stress-aware algorithm for power and temperature management of mpsocs," in Proceedings of the 2015 Design, Automation \& Test in Europe Conference \& Exhibition. EDA Consortium, 2015, pp. 954-959.

[18] T. E. Carlson, W. Heirman, and L. Eeckhout, "Sniper: Exploring the level of abstraction for scalable and accurate parallel multi-core simulations," in International Conference for High Performance Computing, Networking, Storage and Analysis (SC), Nov. 2011, pp. 52:1-52:12.

[19] G. Singla, G. Kaur, A. K. Unver, and U. Y. Ogras, "Predictive dynamic thermal and power management for heterogeneous mobile platforms," in Proceedings of the 2015 Design, Automation \& Test in Europe Conference \& Exhibition. EDA Consortium, 2015, pp. 960-965.

[20] G. Bhat, G. Singla, A. K. Unver, and U. Y. Ogras, "Algorithmic imization of thermal and power management for heterogeneous mobile platforms," IEEE Transactions on Very Large Scale Integration (VLSI) Systems, vol. 26, no. 3, pp. 544-557, 2018.

[21] B. K. Reddy et al., "Inter-cluster thread-to-core mapping and dvfs on heterogeneous multi-cores," IEEE Transactions on Multiscale Computing Systems, pp. 1-14, 2017.

[22] S. Dey, A. K. Singh, D. K. Prasad, and K. D. Mcdonald-Maier, "Socodecnn: Program source code for visual cnn classification using computer vision methodology," IEEE Access, vol. 7, pp. $157158-$ $157172,2019$.

[23] D. Opitz and R. Maclin, "Popular ensemble methods: An empirical study," Journal of artificial intelligence research, vol. 11, pp. 169-198, 1999.

[24] K. Anand et al., "A compiler-level intermediate representation based binary analysis and rewriting system," in Proceedings of the 8th ACM European Conference on Computer Systems. ACM, 2013.

[25] S. J. Pan et al., "A survey on transfer learning," IEEE Transactions on knowledge and data engineering, vol. 22, no. 10, pp. 1345-1359, 2010.

[26] M. D. Zeiler and R. Fergus, "Visualizing and understanding convolutional networks," in European conference on computer vision. Springer, 2014, pp. 818-833.

[27] "Exynos 5 octa (5422)," https://www.samsung.com/exynos, accessed: 2018-07-23.

[28] “Arm big.little technology," http://www.arm.com/, accessed: 2018-07-23.

[29] "Odroid smartpower2," https://www.hardkernel.com/shop/smartpower2with-15v-4a/, accessed: 2020-07-07.

[30] C. Bienia, "Benchmarking modern multiprocessors," Ph.D. dissertation, Princeton University, January 2011.

[31] S. Soo, "Object detection using haar-cascade classifier," Institute of Computer Science, University of Tartu, pp. 1-12, 2014.

[32] H. J. Curnow and B. A. Wichmann, "A synthetic benchmark," The Computer Journal, vol. 19, no. 1, pp. 43-49, 1976. 